\title{
Die meistzitierten Arbeiten des 40. Jahrgangs der Psychiatrischen Praxis - Herzlichen Glückwunsch!
}

Autoren

Institut
Sonja Kolbe, Steffi G. Riedel-Heller

Institut für Sozialmedizin, Arbeitsmedizin und Public Health (ISAP), Medizinische Fakultät, Universität Leipzig

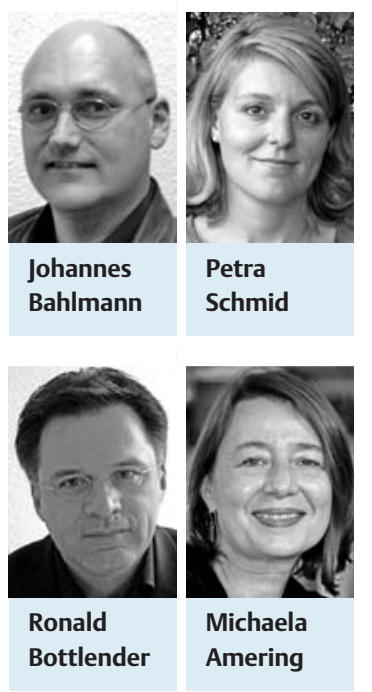

Bibliografie

Dol http://dx.doi.org/ 10.1055/s-0041-110089

Psychiat Prax 2016; 43: 7

(c) Georg Thieme Verlag KG

Stuttgart · New York

ISSN 0303-4259

\section{Korrespondenzadresse}

Prof. Dr. med.

Steffi G. Riedel-Heller, MPH

Direktorin, Institut für

Sozialmedizin, Arbeitsmedizin und Public Health (ISAP),

Universität Leipzig,

Medizinische Fakultät

Philipp-Rosenthal-Straße 55

04103 Leipzig

Steffi.Riedel-Heller@medizin.

uni-leipzig.de

\section{Stigma}

$\nabla$

Als meistzitierte Publikation küren wir eine Originalarbeit von Dipl.-Psych. Johannes Bahlmann, Psychologischer Psychotherapeut in der Klinik für Psychiatrie und Psychotherapie der Universitätsmedizin Greifswald am HELIOS-Hanse-Klinikum Stralsund, für seine Arbeit zur Frage, ob die Bezeichnung „Burnout“ in der Allgemeinbevölkerung mehr Akzeptanz findet als die Bezeichnung „Depression“ und welche Folgen dies für die Behandlungsempfehlungen hat [1]. Durch eine repräsentative Bevölkerungsumfrage konnten er und seine Kollegen zeigen, dass die Bezeichnung „Burnout“ in der sozialen Akzeptanz leichte Vorteile hat, jedoch deutliche Nachteile bei den Behandlungsempfehlungen mit sich bringt. Sollte also der Begriff „Burnout“ in Zukunft doch gut überlegt und weniger oft verwendet werden?

\section{Integrierte Versorgung \\ $\nabla$}

Auf dem zweiten Platz folgt eine Übersichtsarbeit von Dr. Dipl.-Psych. Petra Schmid, Versorgungsforscherin und therapeutische Leiterin der Suchtaufnahmestation des Zentrums für Psychiatrie Südwürttembergs Ravensburg-Weissenau. Die Übersicht widmet sich dem Implementierungsstand und der Begleitevaluation der sektorübergreifenden Versorgung in Deutschland [2]. Sektorübergreifende Modelle (z.B. Regionalbudget, integrierte Versorgung) finden zunehmend Eingang in die psychiatrische Versorgung, sind allerdings sehr heterogen und zeigen bezüglich der Leistungsnutzung ein uneinheitliches Bild. Erfahrungen aus Modellprojekten sind wegweisend und unverzichtbar.

\section{Recovery $\nabla$}

Ist „Recovery“ eine Illusion? Diese Frage stellten sich PD Dr. med. Ronald Bottlender (Direktor der Klinik für Psychiatrie und Psychotherapie, Klinikum Lüdenscheid/Akademisches Lehrkrankenhaus der Universität Bonn) und Prof. Dr. Michaela Amering (Oberärztin an der Universitätsklinik für
Psychiatrie und Psychotherapie, Universität Wien) in unserer meistzitierten Debatte [3]. „Ja! “ sagt Herr Bottlender, er findet den Recovery-Begriff zu unscharf definiert und sieht Recovery auch eher als „alten Wein in neuen Schläuchen“. Dagegen hält Frau Amering, dass sicherlich gerade in Punkten wie Stigmatisierung psychisch Kranker, Teilhabe am sozialen Leben und Ähnlichem noch ein erheblicher Bedarf an Veränderung besteht. Deshalb sei Recovery aber keinesfalls gescheitert oder illusorisch, sondern sollte eher Ansporn sein, um auch diese Hürden noch zu meistern. Die Debattanten haben mit ihrem akademischen Duell, das sich einem wichtigen und zukunftsträchtigen Thema widmet, die meisten LeserInnen erreicht!

\section{Ausblick \\ $\nabla$}

Die Themen der meistzitierten Arbeiten sind relevant für die Psychiatrische Praxis, was sich auch in ganz aktuellen Publikationen dazu zeigt. So stellten sich AutorInnen im letzten Jahr beispielsweise die Frage, wie der „Trialog“ von psychisch Kranken, Angehörigen und Professionellen erlebt wird [4] und wie Aufklärung, Wissen und Stigmatisierungen von psychischen Erkrankungen verbessert werden können [5].

Wir gratulieren den Autorinnen und Autoren ganz herzlich!

\section{Literatur}

1 Bahlmann J, Angermeyer MC, Schomerus G. „Burnout“ statt „Depression“ - eine Strategie zur Vermeidung von Stigma? Psychiat Prax 2013; 40: 78-82

2 Schmid P, Steinert T, Borbé R. Systematische Literaturübersicht zur Implementierung der sektorübergreifenden Versorgung (Regionalbudget, integrierte Versorgung) in Deutschland. Psychiat Prax 2013; 40: 414-424

3 Bottlender R, Amering M. Recovery ist eine Illusion Pro \& Kontra. Psychiat Prax 2013; 40: 244-247

4 von Peter S, Schwedler HJ, Amering M et al. „Diese Offenheit muss weitergehen" - Wie erleben Psychiatrieerfahrene, Angehörige und Professionelle den Trialog? Psychiat Prax 2015; 42: 384-391

5 Lambert M, Härter M, Arnold D et al. Verbesserung von Aufklärung, Wissen und Stigmatisierung psychischer Erkrankungen in der Hamburger Bevölkerung. Psychiat Prax 2015; 42 (Suppl. 01): S9-S13 